\title{
A small intestinal organoid model of non-invasive enteric pathogen-epithelial cell interactions
}

\author{
SS Wilson ${ }^{1}$, A Tocchi ${ }^{2}$, MK Holly ${ }^{1}$, WC Parks ${ }^{2,3,4}$ and JG Smith ${ }^{1}$
}

Organoids mirror in vivo tissue organization and are powerful tools to investigate the development and cell biology of the small intestine. However, their application for the study of host-pathogen interactions has been largely unexplored. We have established a model using microinjection of organoids to mimic enteric infection, allowing for direct examination of pathogen interactions with primary epithelial cells in the absence of confounding variables introduced by immune cells or the commensal microbiota. We investigated the impact of Paneth cell $\alpha$-defensin antimicrobial peptides on bacterial growth. We demonstrate that organoids form a sealed lumen, which contains concentrations of $\alpha$-defensins capable of restricting growth of multiple strains of Salmonella enterica serovar Typhimurium for at least $20 \mathrm{~h}$ postinfection.

Transgenic expression of human defensin 5 in mouse organoids lacking functional murine $\alpha$-defensins partially restored bacterial killing. We also found that organoids from NOD2 ${ }^{-l-}$ mice were not impaired in $\alpha$-defensin expression or antibacterial activity. This model is optimized for the study of non-invasive bacteria but can be extended to other enteric pathogens and is amenable to further genetic manipulation of both the host and microbe to dissect this critical interface of host defense.

\section{INTRODUCTION}

The complexity of the small intestinal epithelium has been difficult to model in culture, and dissection of epitheliumspecific functions in vivo is confounded by the influence of signals arising from local and systemic non-epithelial sources. The discovery of a system to culture primary stem cell-derived small intestinal organoids has addressed these issues: First, a defined growth medium allows for the differentiation and maintenance of goblet cells, Paneth cells, enterocytes, enteroendocrine cells, and stem cells that recapitulates the cellular composition of the small intestinal epithelium. ${ }^{1,2}$ Second, organoids have crypt-like domains and villus-like regions and can be stably maintained in sterile culture in the absence of mesenchymal and immune cells. Third, artifacts of transformation inherent in most traditional intestinal epithelial culture models are absent. Organoids have been increasingly used to uncover aspects of cell biology, intestinal development, and wound repair but have not been widely utilized to study pathogen-epithelial cell interactions. ${ }^{1,3}$ To this end, we have developed a new model of enteric infection by accessing the apical aspect of the polarized intestinal epithelium of organoids using microinjection.

As proof of concept, we used our new model to assess the need for $\alpha$-defensins in epithelial defense against a replicating bacterial pathogen, Salmonella enterica serovar Typhimurium (STM). $\alpha$-Defensins are small, cationic, amphipathic peptides that possess broad anti-microbial activity; however, there have been limited studies of the direct anti-microbial activity of naturally secreted $\alpha$-defensins in a complex, physiological extracellular milieu. ${ }^{4}$ Moreover, their role in modulating bacterial pathogenesis in vivo is confounded by indirect effects of $\alpha$-defensins on the immune system and the composition of the commensal microbiota. ${ }^{5-7}$

To address these shortcomings, our enteric infection model enables the first direct assessment of STM killing during prolonged ex vivo co-culture with viable primary epithelial cells capable of naturally producing enteric $\alpha$-defensins. For this purpose, we established small intestinal organoids from wildtype (WT) and $M m p 7^{-1-}$ mice. As matrix metalloproteinase 7 (MMP7, matrilysin) is the protease that converts mouse

\footnotetext{
${ }^{1}$ Department of Microbiology, University of Washington, Seattle, Washington, USA. ${ }^{2}$ Department of Pathology, University of Washington, Seattle, Washington, USA and ${ }^{3}$ Department of Medicine, University of Washington, Seattle, Washington, USA. Correspondence: JG Smith (jgsmith2@uw.edu)

${ }^{4}$ Current address: Cedars-Sinai Medical Center, Los Angeles, California 90048, USA.
} 
pro- $\alpha$-defensins into their mature forms, $M m p 7^{-1-}$ mice lack functional $\alpha$-defensins in the small intestine. ${ }^{8}$ Through comparative assays using these two genotypes, we have shown by two approaches that $\alpha$-defensins substantially contribute to epithelial host defense and restrict growth of STM for at least $20 \mathrm{~h}$ in culture. Growth inhibition was seen for multiple strains of STM and at several time points postinjection. The assay is also responsive to host factors influencing Paneth cell function, as transgenic expression of human defensin 5 (HD5) in $M m p 7^{-1-}$ organoids restored bacterial killing. Nonetheless, although nucleotide binding and oligomerization domain 2 (NOD2) deficiency has been linked to reduced $\alpha$-defensin expression and function in the etiology of Crohn's disease, ${ }^{9}$ we found that bacterial killing in organoids from NOD2 $2^{-1-}$ mice was not impaired and that $\alpha$-defensin expression in these organoids was equivalent to WT. In summary, we have created and validated a novel model to investigate interactions between enteric pathogens and small intestinal epithelial cells that can be extended to additional bacterial and viral pathogens and can be genetically dissected at both the host and pathogen level.

\section{RESULTS}

\section{The organoid lumen is intact and can be accessed by microinjection}

For organoids to accurately model the intestine, their lumen and the apical surface of the cells should be sequestered from the extracellular basolateral environment. An intact organoid lumen would potentially allow for the accumulation of a high local concentration of secretion products, including $\alpha$-defensins. To access this space and mimic apical enteric infection, we utilized microinjection. We established primary small intestinal organoid cultures from WT C57BL/6 mice. ${ }^{1,10}$ Microinjected phosphate-buffered saline (PBS) caused swelling of organoids, indicating the integrity of the lumen (Figure 1a). To demonstrate further that the lumen was functionally intact, we microinjected $\sim 5 \times 10^{4}$ colony-forming units (CFU)/ organoid of the non-invasive STM strain LT2 $\triangle$ phoP either into the organoid lumen (inside) or in the surrounding Matrigel (outside) proximal to organoids. All samples received exactly 20 injections in total. We then incubated the cultures for $2 \mathrm{~h}$, treated with or without $100 \mu \mathrm{g} \mathrm{ml}^{-1}$ of the cell membraneimpermeant antibiotic gentamicin for $2 \mathrm{~h}$ and enumerated surviving $\mathrm{CFU}$. We recovered equal numbers of $\mathrm{CFU}$ from inside and outside of the organoids in the absence of gentamicin; however, $>10^{3}$ CFU were protected from gentamicin when injected inside the organoids (Figure 1b).

Enteric $\alpha$-defensins are found in secretory granules of Paneth cells in the small intestine. ${ }^{11,12}$ Unlike humans, mice express an expanded repertoire of enteric $\alpha$-defensins, termed cryptdins. ${ }^{13}$ Mouse $\alpha$-defensins are produced as pro-peptides and cleaved and activated to their mature form by MMP7 in Paneth cells. ${ }^{8}$ Accordingly, $M m p 7^{-/-}$mice have normal expression of pro-defensins in their Paneth cell granules but are functional knockouts of mature $\alpha$-defensins in the small intestine. ${ }^{8,14}$ We established organoids from $M m p 7^{-/-}$ mice (C57BL/6) and found that absence of MMP7 did not alter
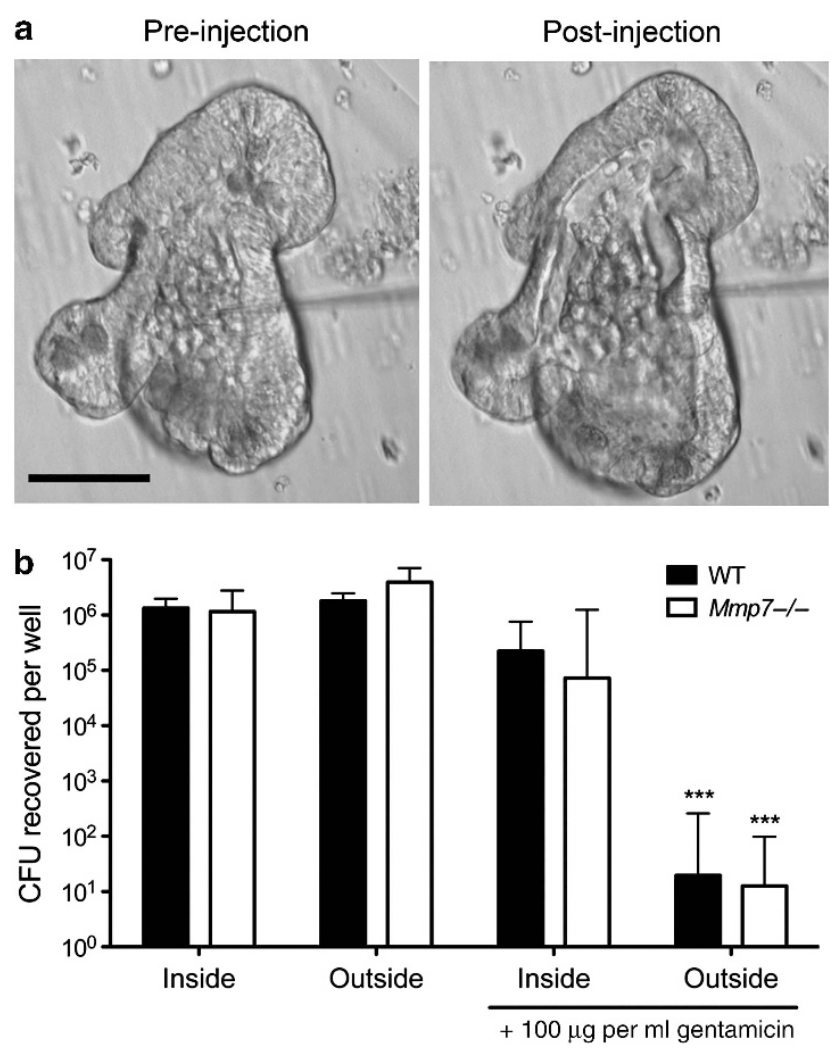

Figure 1 The organoid lumen is intact and can be accessed by microinjection. (a) Before and after images of microinjection of phosphatebuffered saline into the organoid lumen. Bar $=50 \mu \mathrm{m}$. (b) Survival of Salmonella enterica serovar Typhimurium after injection into the organoid lumen (inside) or into the surrounding Matrigel (outside) in the presence and absence of $100 \mu \mathrm{g} \mathrm{ml}^{-1}$ gentamicin. Data for wild-type (WT, black bars) and $M m p 7^{-1-}$ (white bars) organoids are the antilog of the average of log-transformed colony-forming units (CFU) from three independent experiments \pm s.d. For statistical significance, one-way analysis of variance with Tukey's post-tests was used to compare all pairs of columns. Only the indicated bars $\left({ }^{\star \star *} P<0.005\right)$ were significant, and they were significant in comparison with every other condition except each other.

the integrity of the organoid lumen (Figure 1b). Overall, these data show that the organoid lumen is intact and can be accessed via microinjection.

\section{Bacterial growth is inhibited in WT but not in $M m p 7^{-1-}$ organoids}

We next asked whether $\alpha$-defensins present in the organoid lumen could inhibit STM growth. WT and $M m p 7^{-1-}$ organoids were injected with STM expressing green fluorescent protein (GFP) (LT2 $\triangle$ phoP GFP, $5 \times 10^{3}$ CFU/organoid) and imaged at 0 and $5 \mathrm{~h}$ postinjection. In three separate experiments, a reduction in fluorescence occurred by $5 \mathrm{~h}$ postinjection in WT organoids but not in $M m p 7^{-1-}$ organoids (Figure 2a-c). Rather, diffusion of the bacteria within the organoid lumen from the site of injection was seen in $M m p 7^{-1-}$ organoids. The surviving CFU were quantified at $20 \mathrm{~h}$ postinjection, and a 3.9-log reduction in CFU was seen in WT organoids compared with $M m p 7^{-1-}$ organoids (Figure 2d). To correlate quantification of bacterial killing by fluorescence and CFU, 
a
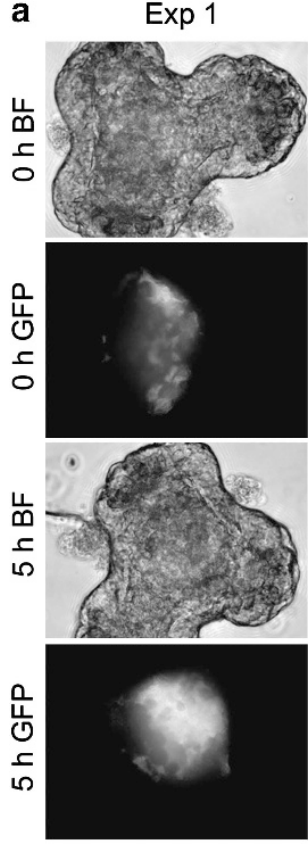

C

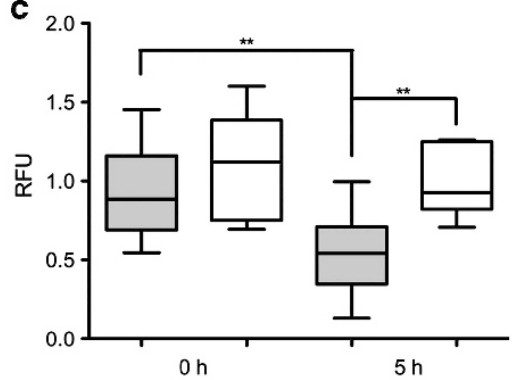

Exp 2
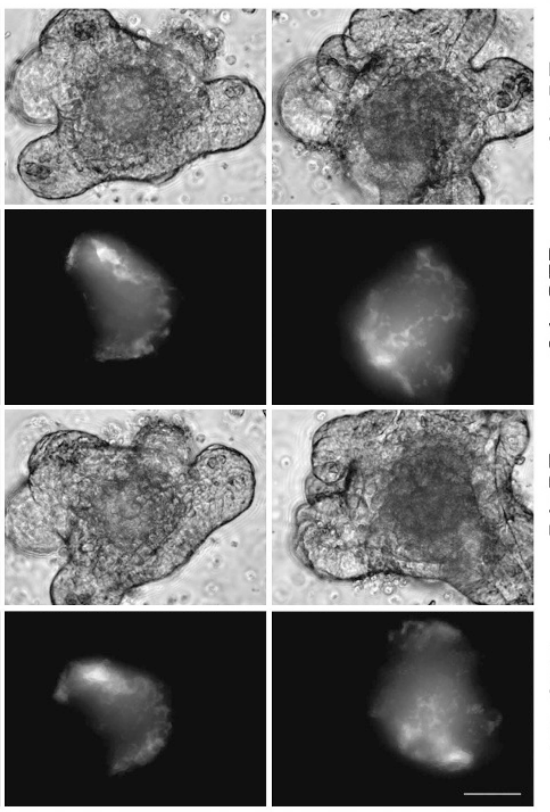

b

b Exp 1
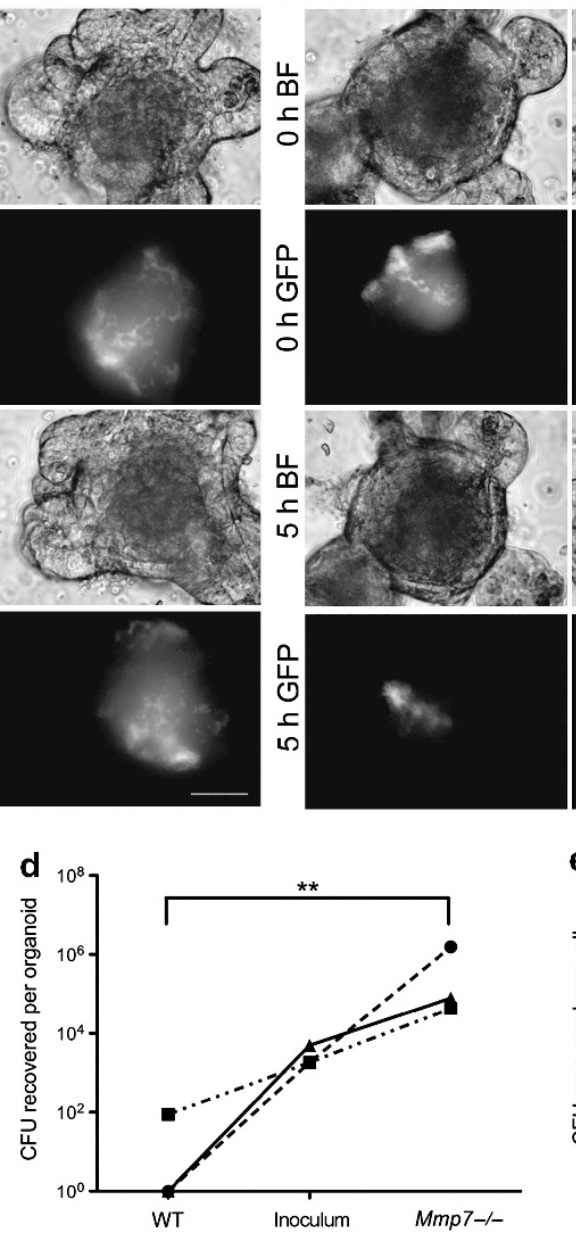

Exp 2

Exp 3
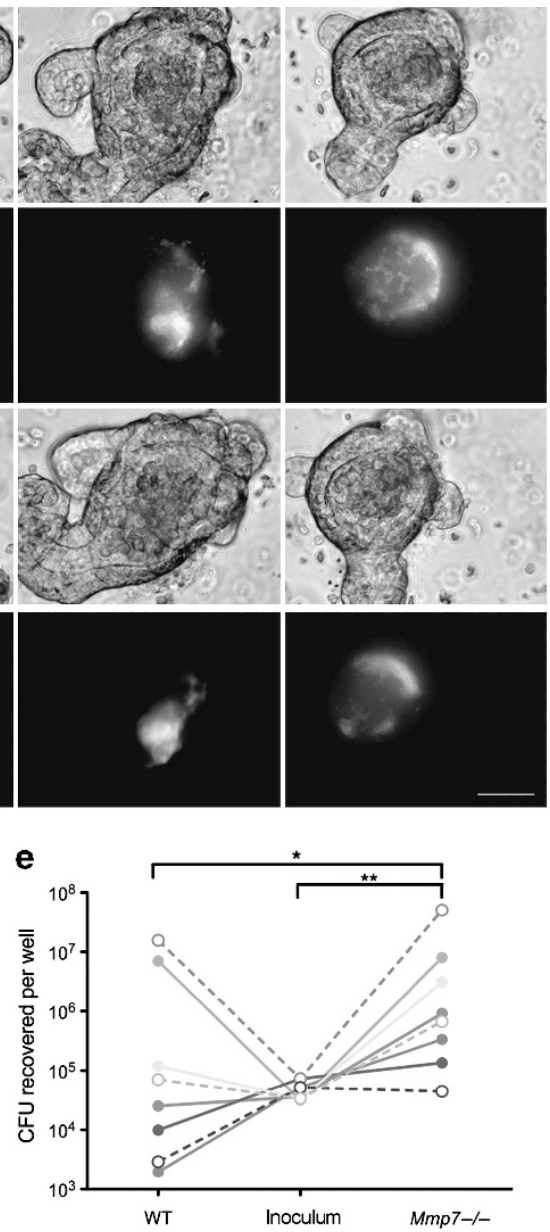

Figure 2 Bacterial growth is inhibited in wild-type (WT) but not $M m p 7^{-1-}$ organoids. Representative images from three independent experiments of Salmonella enterica serovar Typhimurium LT2 $\triangle$ phoP green fluorescent protein (GFP) microinjected into (a) Mmp $7^{-1-}$ organoids and (b) WT organoids. Images were taken at 0 and $5 \mathrm{~h}$ postinjection, and the bright field (BF) image corresponds to the $0 \mathrm{~h}$ GFP image. Signal above threshold is shown for each image. (c) Data are the relative fluorescence intensity (RFU) of three injected organoids per experiment (nine total) for WT (grey) and $M m p 7^{-/-}$(white). Whiskers are the minimum and maximum of the data, and the horizontal line is the mean. (d) Colony-forming units (CFU) recovered $20 \mathrm{~h}$ postinjection. Each line corresponds to one experiment in panels $\mathbf{a}$ and $\mathbf{b}$, and each data point is the average CFU from two to three organoids. (e) CFU recovered $9 \mathrm{~h}$ postinjection. Each line represents one independent experiment, and each data point is the total CFU from 20 organoids injected in a single well. Dashed lines with open symbols represent experiments using organoids pretreated with $10 \mu \mathrm{m}$ carbamylcholine chloride. Bars $=50 \mu \mathrm{m}(\mathbf{a}$ and $\mathbf{b})$. ${ }^{*} P<0.05$, ${ }^{\star *} P<0.01$.

we imaged and then immediately isolated bacteria from parallel cultures of WT and $M m p 7^{-1-}$ organoids at 0, 5, and $9 \mathrm{~h}$ postinfection. Under these conditions we found a close correlation between the two assays with a $1.3-\log$ reduction in survival and a 1-log reduction in average fluorescence intensity in WT compared with $M m p 7^{-1-}$ at $5 \mathrm{~h}$ postinjection that increased to a 1.9-log reduction in survival and a 1.3-log reduction in average fluorescence intensity in WT compared with $M m p 7^{-1-}$ at $9 \mathrm{~h}$ (see Supplementary Figure S1 online). These results indicate that changes in CFU over this time period reflect bacterial dynamics in the lumen.

We then assessed the magnitude of $\alpha$-defensin-mediated killing in organoids from multiple independent preparations. WT and $M m p 7^{-1-}$ organoids were injected with LT2 $\triangle p h o P$ $\left(5 \times 10^{3} \mathrm{CFU} /\right.$ organoid), and surviving $\mathrm{CFU}$ from 20 injected organoids were pooled and assayed at $9 \mathrm{~h}$ postinjection. We observed a 1.1-log reduction in WT organoids compared with $M m p 7^{-1-}$ organoids (Figure 2e). In addition, CFU in the $M m p 7^{-1-}$ organoids increased in relation to the inoculum, indicating that the bacteria were able to grow in the organoid lumen (Figure 2e). We then examined the effect of carbamylcholine chloride, a known stimulator of Paneth cell secretion, ${ }^{4}$ and found that although carbamylcholine chloride induced organoid swelling, indicative of an effect on secretion (data not shown), it did not alter the magnitude of bacterial killing (dashed lines in Figure 2e). Taken together, these results by two distinct measures support the conclusion that STM were viable in the organoid lumen only in the absence of functional $\alpha$-defensins.

Organoids from $M m p 7^{-1-}$ mice lack mature $\alpha$-defensins To support our hypothesis that impaired STM killing in $M m p 7^{-1-}$ organoids was due to a specific absence of 


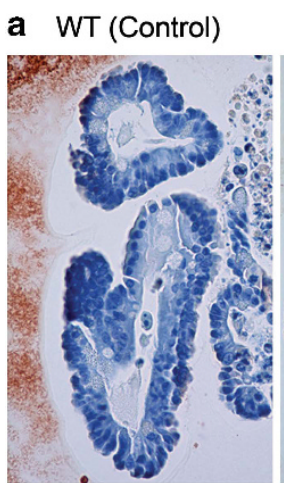

WT ( $\alpha$-Crp5)

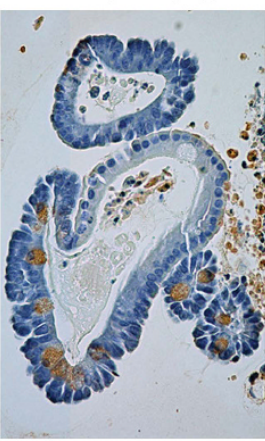

b

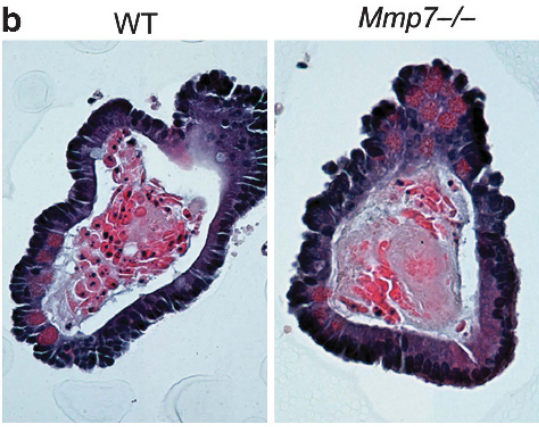

d

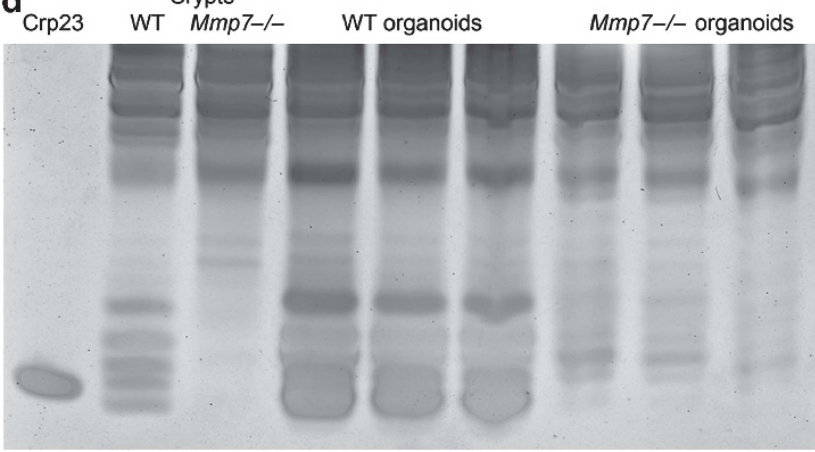

Figure 3 Organoids from $M m p 7^{-1-}$ mice lack mature $\alpha$-defensins. (a) Immunohistochemistry of wild-type (WT) and $M m p 7^{-/-}$organoids stained with goat anti-Crp5 antibody or control goat immunoglobulin G. (b) WT and $\mathrm{Mmp7}^{-1}$ organoids stained with hematoxylin and eosin. (c) Immunoblots of lysates from WT and $M m p 7^{-1-}$ organoids probed for matrix metalloproteinase 7 (MMP7). Recombinant MMP7 (rMMP7) was used as a positive control, and an antibody to GAPDH (glyceraldehyde 3-phosphate dehydrogenase) was used as a control for loading. The upper band in the rMMP7 lane corresponds to pro-MMP7 (30 kDa), and the lower band is the active form $(20 \mathrm{kDa})$. The mobilities of active rMMP7 and active MMP7 in the lysates differ due to the presence of additional mass from the purification tag. (d) Equal amounts $(100 \mu \mathrm{g})$ of lyophilized extract from freshly isolated crypts or organoids from WT and $M m p 7^{-1-}$ mice were analyzed by acid-urea polyacrylamide gel electrophoresis. Each lane of organoid extract is from a separate preparation of organoids from different mice. Purified mouse $\alpha$-defensin-23 (Crp23, $1 \mu \mathrm{g})$ indicates the mobility of mature $\alpha$-defensins.

functional $\alpha$-defensins, we assessed the relative levels of proand mature $\alpha$-defensins in WT and $M m p 7^{-1-}$ organoids. Organoids from both genotypes grew at similar rates and contained equivalent proportions of goblet and Paneth cells, which were readily apparent (Figure $\mathbf{3 a}, \mathbf{b}$ and see Supplementary Figure S2A-C). To confirm the production of pro- $\alpha$-defensins, we stained organoids from both mouse strains for mouse $\alpha$-defensin-5 (Crp5). We observed specific staining for Crp5 in the Paneth cells of both WT and $M m p 7^{-1-}$ organoids. No staining was observed with the control antibody (Figure 3a). Thus, Paneth cells in organoids from both WT and $M m p 7^{-1-}$ mice produce granules containing pro- $\alpha$-defensins.

Next, the presence and activation state of $\alpha$-defensins in WT and $M m p 7^{-1-}$ organoids were directly assessed by acid-urea polyacrylamide gel electrophoresis (AU-PAGE). Equivalent amounts of total protein extracted from WT and $M m p 7^{-1-}$ organoids were compared with extracts of crypt-enriched fractions from WT and $M m p 7^{-1-}$ mice. Purified Crp23, an abundant $\alpha$-defensin expressed in C57BL/6 mice, was included as a control. ${ }^{13}$ Extracts from freshly isolated crypts and organoids derived from WT mice contained species with mobilities consistent with mature $\alpha$-defensins. In contrast, extracts from $\mathrm{Mmp7}^{-1-}$ crypts and organoids did not (Figure 3d). Furthermore, abundant active MMP7, which is present in Paneth cell granules in vivo and responsible for activation of pro- $\alpha$-defensins, ${ }^{8}$ was produced in WT but absent from $M m p 7^{-1-}$ organoids (Figure 3c). These findings provide further evidence that organoids recapitulate the state of $\alpha$-defensin maturation reported for WT and $M m p 7^{-1-}$ mice. $^{14}$

\section{Increased sensitivity of $\triangle$ phoP STM mutants confirms a role} for $\alpha$-defensins in luminal killing

To further support the critical and specific role of $\alpha$-defensinmediated bacterial killing in this model, we compared the survival of WT and LT2 $\triangle p h o P$ in organoids from both genotypes. The STM phoP phoQ two-component regulatory system is critical for sensing and responding to antimicrobial peptides, and STM phoP mutants are more sensitive to purified $\alpha$-defensins. ${ }^{15}$ For these experiments, we chose a later time point (20 $\mathrm{h}$ postinjection) to amplify any survival difference. A dose-response study identified $50 \mathrm{CFU} /$ organoid as a minimal dose that reliably yielded quantifiable surviving CFU (data not shown). Significant bacterial killing was observed for both strains in WT organoids compared with $M m p 7^{-1-}$ organoids (Figure 4a). Consistent with the role of phoP in sensitizing STM to $\alpha$-defensins, the difference was greater in LT2 $\triangle p h o P$ (4.6-log reduction) than in WT LT2 (1.7-log reduction).

Next, we assessed the ability of organoids to defend against STM 14028s, a strain commonly used in mouse models of Salmonella pathogenesis. ${ }^{8}$ We introduced a chromosomal sipB mutation that renders the bacteria unable to attach to and therefore invade epithelial cells and assessed the impact of phoP. ${ }^{16}$ After $20 \mathrm{~h}$, we observed a 6-log reduction in CFU in the 14028s $\triangle p h o P$ background but not in the WT strain (Figure $4 \mathbf{b}$ ). Thus the increased killing of the $\triangle p h o P$ mutants of both STM strains confirms the importance of $\alpha$-defensins in mediating killing in our model.

\section{Transgenic expression of HD5 rescues bacterial killing} Having established the importance of bacterial genotype in $\alpha$-defensin-mediated killing in our system, we sought to 

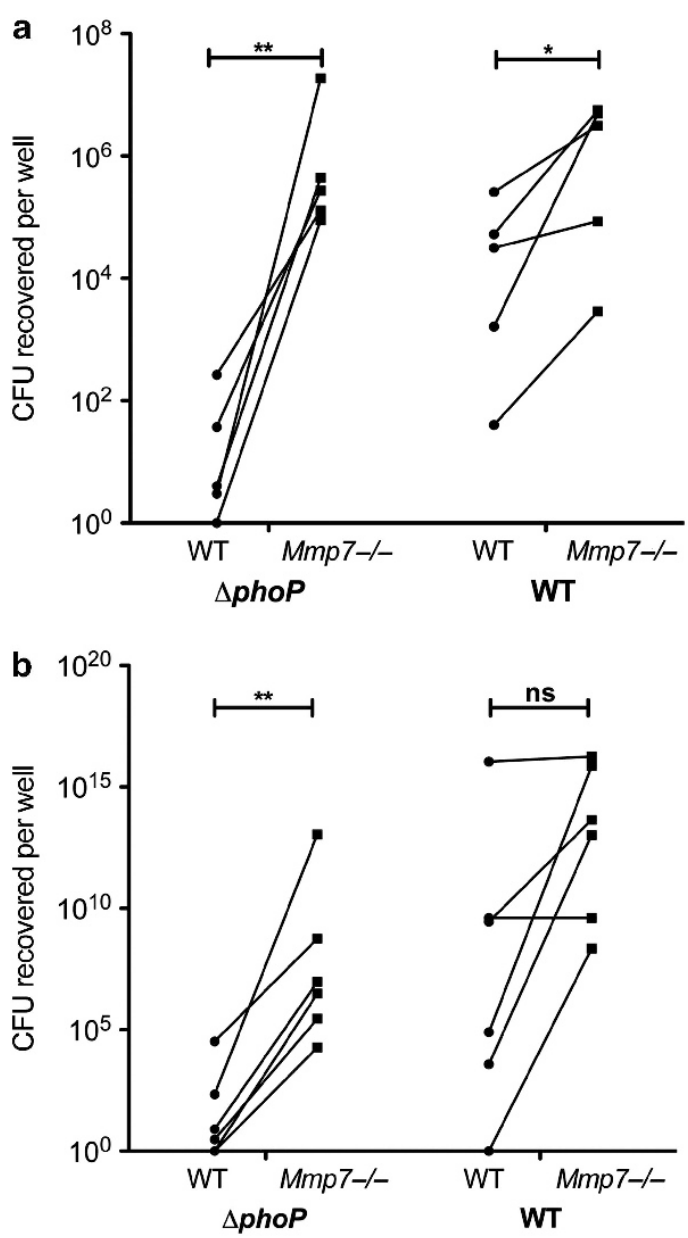

Figure 4 Long-term killing of multiple Salmonella enterica serovar Typhimurium (STM) strains. (a) STM LT2 and STM LT2 $\triangle$ phoP or (b) $14028 \mathrm{~s} \triangle \operatorname{sip} B$ (wild type (WT)) and $14028 \mathrm{~s} \Delta \operatorname{sip} B \Delta$ phoP were injected into WT and $M m p 7^{-1-}$ organoids, and surviving colony-forming units (CFU) were enumerated $20 \mathrm{~h}$ postinjection. Each line represents an independent paired experiment, and each point is the pooled data from a well of 20 injected organoids. ${ }^{*} P<0.05,{ }^{* \star} P<0.01$. NS, not significant.

measure the effect of additional host genotypes relevant to Paneth cell function. We established organoids from $M m p 7^{-1-}$ mice that express transgenic HD5 $\left(M m p 7^{-1-} D E F A 5^{+/-}\right)$. Mice expressing transgenic HD5 on a WT background $\left(M m p 7^{+/+} D E F A 5^{+/+}\right)$are resistant to oral challenge with STM; however, there has been no investigation into the antibacterial activity of $M m p 7^{-1-} D E F A 5^{+1-}$ Paneth cells. ${ }^{17}$

We first examined the processing of HD5 in organoids. In humans, proHD5 is cleaved by endogenous Paneth cell trypsin; however, trypsin is not present in mouse Paneth cells. ${ }^{18}$ Although HD5 can also be processed by MMP7 in vitro, ${ }^{18}$ mature HD5 has also been shown in vivo in the $M m p 7^{-1-} D E F A 5^{+1-}$ background and has been attributed to alternative host and microbial proteinases. ${ }^{14}$ We observed processed HD5 in organoids and isolated crypts from both $M m p 7^{-1-} D E F A 5^{+1-}$ and $M m p 7^{+/+}$DEFA5 ${ }^{+/+}$mice via western blotting (Figure 5a).

We then asked whether transgenic expression of HD5 augmented Paneth cell anti-bacterial activity. As in our previous assays, $5 \times 10^{3} \mathrm{CFU}$ per organoid of STM LT2 $\triangle p h o P$ was injected into WT, $M m p 7^{-1-}$, and $M m p 7^{-1-}$ $D E F A 5^{+/-}$organoids. Surviving CFU were enumerated $7 \mathrm{~h}$ (Figure 5b) and $16 \mathrm{~h}$ postinjection (Figure $5 \mathrm{c}$ ) to capture the dynamics of STM killing by HD5. After $7 \mathrm{~h}$, survival of the inoculum was reduced 50-fold upon injection into WT organoids. Although differences among genotypes were not significant, we observed the greatest survival in $M m p 7^{-1-}$ organoids and intermediate survival in $M m p 7^{-1-} D E F A 5^{+/-}$ organoids. By $16 \mathrm{~h}$ postinjection, STM grew in organoids from all three genotypes; however, survival between WT and $M m p 7^{-1-}$ (2.1-log difference) was significant. Moreover, the transgenic expression of HD5 restored WT killing in the $M m p 7^{-1-}$ $D E F A 5^{+/-}$organoids, with a 2.3-log difference in survival between $M m p 7^{-1-}$ and $M m p 7^{-1-} D E F A 5^{+1-}$. Consistent with Supplementary Figure S1, there is an initial decrease in $\mathrm{CFU}$ in all of the organoid genotypes at $7 \mathrm{~h}$, with a larger reduction in organoids that contain mature $\alpha$-defensins. By $16 \mathrm{~h}$, there is growth across the organoid genotypes from the $7 \mathrm{~h}$ time point, which is much larger in the $M m p 7^{-1-}$ background (3-log increase) than in the WT (1.1-log increase) or $M m p 7^{-1-}$ DEFA5 ${ }^{+/-}(1.6-\log$ increase) organoids.

\section{NOD2 deletion has no effect on bacterial killing}

We next addressed the importance of NOD2 expression on the capacity of organoids to impact bacterial survival. NOD2 is an intracellular receptor for peptidoglycan, and loss-of-function mutations in NOD2 are associated with ileal Crohn's disease and correlated with reduced expression of enteric $\alpha$-defensins. ${ }^{9,19}$ In mouse models, NOD2 deficiency is linked to reduced $\alpha$-defensin expression and impaired antibacterial activity of crypt secretions, indicating that decreased $\alpha$-defensin expression might be an inherent phenotype of NOD2 $2^{-1-}$ mice..$^{20-22}$ However, a more recent study demonstrated the levels and activities of $\alpha$-defensins in NOD2 $2^{-1-}$ mice are similar to those in WT mice. ${ }^{23}$

To address these questions, we compared the levels of activated $\alpha$-defensins between organoids from $N O D 2^{-1-}$ and WT mice and found that they were equivalent (Figure 6a). To assess the impact of NOD2 expression on bacterial killing, we injected $5 \times 10^{3} \mathrm{CFU}$ of STM LT2 $\triangle$ phoP into NOD2 $2^{-1-}$ and WT organoids and enumerated surviving CFU at $16 \mathrm{~h}$ postinjection. We saw no significant difference in recovered $\mathrm{CFU}$ between the organoid genotypes (Figure $\mathbf{6 b}$ ). To ensure that NOD2 was fully activated, we repeated these experiments with the addition of muramyl di-peptide to the inoculum. Again we saw no significant difference in recovered CFU between the genotypes (Figure 6c). Thus NOD2 expression had no effect on intrinsic $\alpha$-defensin levels or Paneth cell antibacterial function in this model, although the magnitude of killing in these C57BL/6J organoids was weaker than that of C57BL/NHsd organoids in our other experiments.

\section{DISCUSSION}

We have established a model to mimic apical enteric infection that utilizes microinjection of small intestinal organoids. 

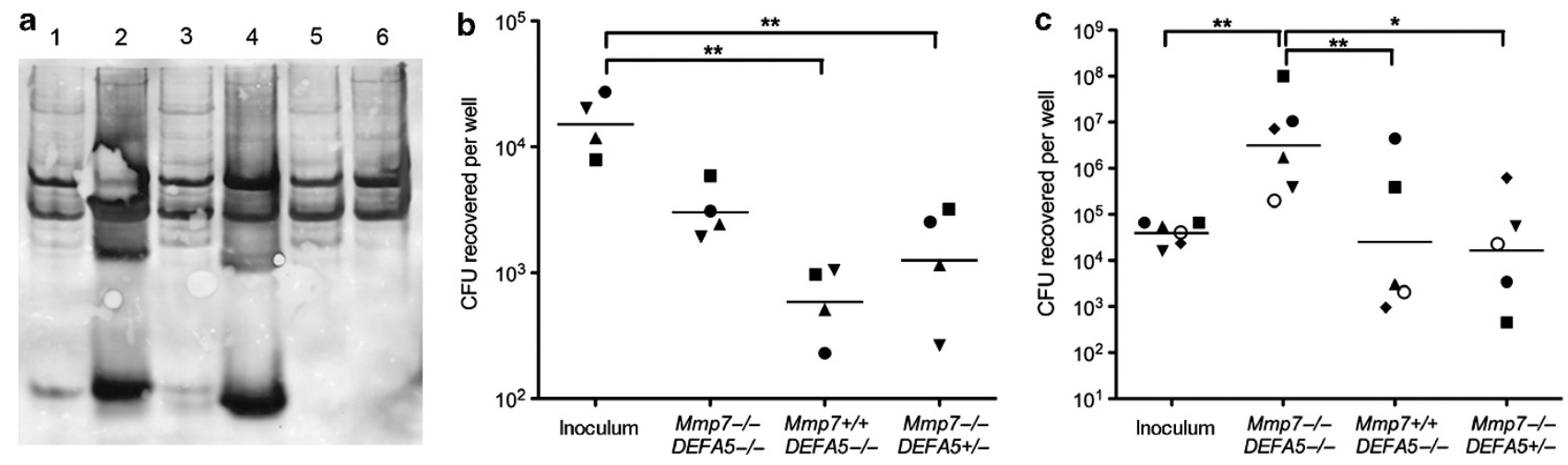

Figure 5 Human defensin 5 (HD5) maturation and Salmonella enterica serovar Typhimurium (STM) killing in $M m p 7^{-1-} D E F A 5^{+/-}$organoids. (a) Acid-urea polyacrylamide gel electrophoresis immunoblot of extracts from $M m p 7^{-1-} D E F A 5^{+/-}$organoids (lane $1,500 \mu \mathrm{g}$ protein) and freshly isolated crypts (lane 2, $250 \mu \mathrm{g}$ protein), $M m p 7^{+/+} D E F A 5^{+/+}$organoids (lane 3, $500 \mu \mathrm{g}$ protein) and freshly isolated crypts (lane 4, $250 \mu \mathrm{g}$ protein), and wild-type (WT) organoids (lane 5, $500 \mu \mathrm{g}$ protein) and freshly isolated crypts (lane 6, $250 \mu \mathrm{g}$ protein) were probed for HD5. (b and c) STM LT2 $\triangle$ phoP was injected into WT, $M m p 7^{-1-}$ and $M m p 7^{-1-} D E F A 5^{+1-}$ organoids, and surviving colony-forming units (CFU) were enumerated $7 \mathrm{~h}(\mathbf{b})$ and $16 \mathrm{~h}(\mathbf{c})$ postinjection. Each symbol represents an independent paired experiment and is the pooled data from a well of 20 injected organoids. ${ }^{\star} P<0.05$, ${ }^{\star *} P<0.01$.

Prominent features of this model include an intact lumen that prevents diffusion of apically secreted products into the bulk volume of the culture medium and the ability to selectively infect either the apical or basolateral aspect of the polarized epithelium. Thus this model allows for a broad range of studies of the interactions between microbes, epithelial cells, and their secreted products. As proof of concept, we determined the impact of naturally secreted $\alpha$-defensins on bacterial survival in the organoid lumen. Growth of non-invasive STM was significantly reduced in WT organoids compared with $M m p 7^{-1-}$ organoids. Killing was observed at multiple time points after injection, by two independent measures, and for two STM strains. In accordance with the survival data, organoids from WT mice produced mature $\alpha$-defensins, whereas those from $M m p 7^{-1-}$ mice did not. In addition, the decreased relative survival of strains lacking the phoP regulon and the ability of transgenic expression of HD5 to restore killing are consistent with an $\alpha$-defensin-dependent mechanism. These results indicate that naturally secreted $\alpha$-defensins in the complex extracellular milieu of the organoid lumen are capable of killing bacteria and that $\alpha$-defensin activity is sustained, protecting the epithelium of the organoid over time.

This model represents a significant improvement over standard methods to determine anti-bacterial activities of $\alpha$-defensins and can be easily applied to studies of other epithelial products. Standard assays for determining the antibacterial activities of $\alpha$-defensins predominately use purified peptides and are performed under low-salt conditions, as bacterial killing is generally inhibited in buffers with physiological levels of salt. ${ }^{24,25}$ A limited number of ex vivo studies have examined the ability of naturally produced $\alpha$-defensins or defensin-containing extracellular fluids to inhibit bacterial growth. ${ }^{25}$ For enteric $\alpha$-defensins, Ayabe et al. ${ }^{4}$ purified crypts or villi from WT and $M m p 7^{-1-}$ mice, incubated them with STM, and enumerated surviving CFU. STM killing was observed after exposure to crypts or crypt secretions but not to villi. In addition, STM killing was markedly reduced in
$M m p 7^{-1-}$ crypts or by an antibody against mouse $\alpha$-defensin- 1 . These data support the notion that secreted $\alpha$-defensins are capable of directly killing bacteria. However, this study utilized freshly isolated small intestinal crypts, which are unstable and only amenable to short-term experiments. Additionally, bacterial killing was demonstrated under low-salt conditions and overall was modest $(<10$-fold) compared with the level of killing we saw with WT organoids (up to a 6-log reduction).

Thus our approach supports the anti-bacterial findings of previous studies with substantial improvements. First, our model enables the study of defensins in a complex milieu without purification and without altering buffer constituents. Second, multiple experiments can be performed with equivalent, stably cultured organoids, which are stable for months to years. In contrast, while ex vivo human and mouse biopsy specimens can be apically infected with bacterial, they are only viable for $12-48$ h. ${ }^{26}$ Third, the fate of bacteria injected into a single organoid can be tracked over time. Fourth, the assay is very sensitive, as it can be amplified through prolonged co-culture of the microbes and organoids and is effective over a wide range of input CFU. Finally, organoids can be genetically manipulated or established from genetically engineered mice, allowing the role of specific proteins and pathways in enteric defense to be assessed. ${ }^{3,27}$

Given these advantages, we applied our enteric infection model to investigate the ability of organoids from NOD2 $2^{-1-}$ mice to inhibit bacterial growth compared with WT organoids. In genome-wide association studies, NOD2 mutations contribute the greatest genetic risk for Crohn's disease, although the exact role that NOD2 mutations have in the etiology of Crohn's disease is unclear. ${ }^{28}$ Initial studies reported a reduction in $\alpha$-defensin transcript levels in NOD2 $2^{-1-}$ mice, which was mirrored in human Crohn's disease patients with NOD2 mutations. ${ }^{9,22}$ This defect was functionally confirmed through a reduction in anti-bacterial activity in $N O D 2^{-1-}$ crypt secretions. ${ }^{20,21}$ However, recent studies have reported WT levels of $\alpha$-defensins in both mice and humans with NOD2 

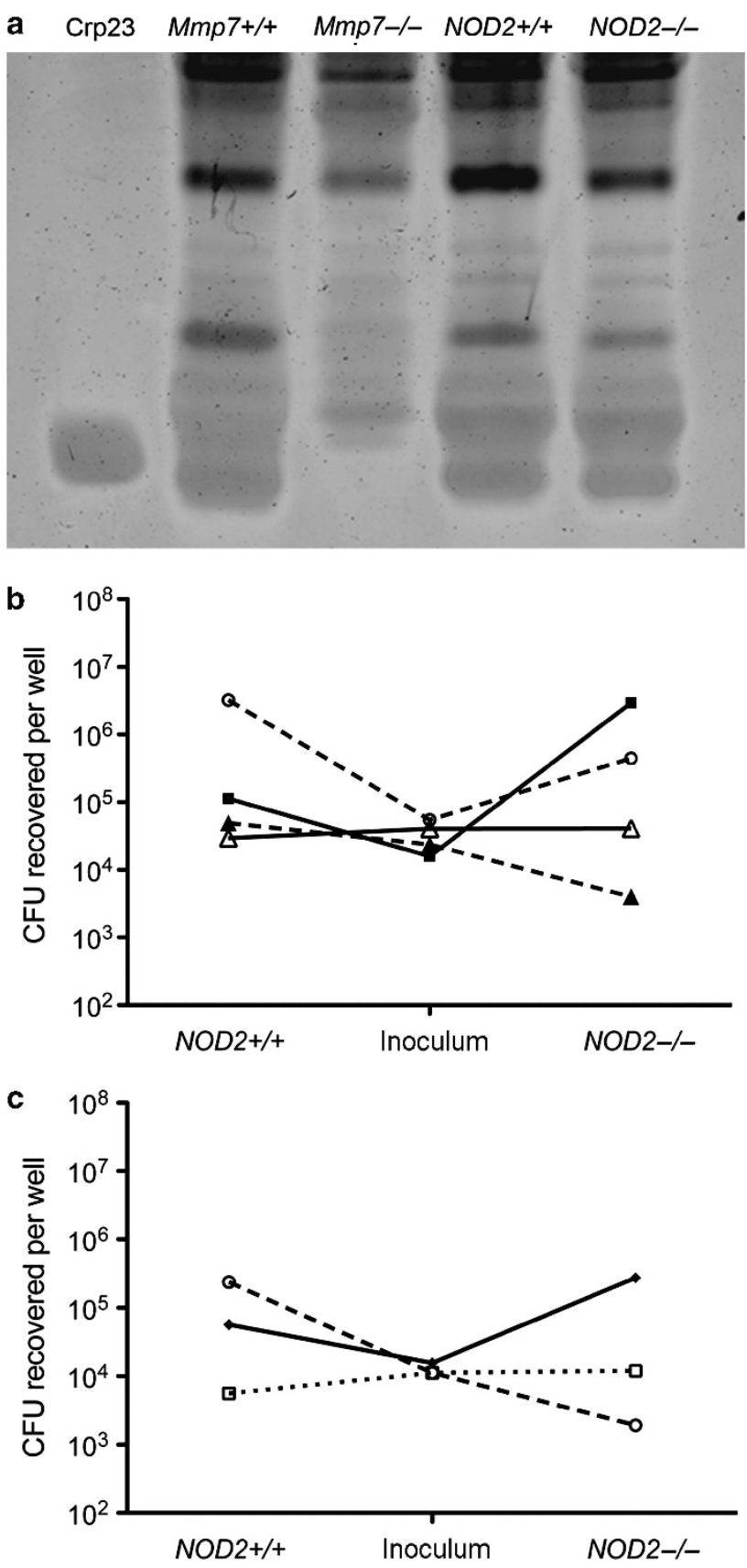

Figure $6 \alpha$-Defensin expression and antibacterial activity of $N O D 2^{-1-}$ organoids. (a) Equal amounts $(100 \mu \mathrm{g})$ of lyophilized extracts from organoids from wild-type, $M m p 7^{-1-}, N O D 2^{+1+}$, and $N O D 2^{-1-}$ mice were analyzed by acid-urea polyacrylamide gel electrophoresis. Purified mouse $\alpha$-defensin$23(\mathrm{Crp23}, 1 \mu \mathrm{g})$ indicates the mobility of mature $\alpha$-defensins. Colonyforming units (CFU) recovered $16 \mathrm{~h}$ postinjection of Salmonella enterica serovar Typhimurium LT2 $\triangle$ phoP mixed without (b) or with (c) $100 \mu \mathrm{g}$ muramyl di-peptide. Each line represents one independent experiment, and each data point is the total CFU from 20 organoids injected in a single well.

deficiency and support a role for NOD2 in stem cell survival. ${ }^{23,29,30}$ We found that NOD2 $2^{-1-}$ organoids contain WT levels of $\alpha$-defensins. Moreover, we observed no defect in their ability to restrict growth of luminal bacteria. These results are most consistent with the absence of an effect of NOD2 on either $\alpha$-defensin expression or Paneth cell function.
Despite the strength of our approach, there are some limitations to the design and interpretation of these and future applications. First, although the epithelial cell diversity of the small intestine is recapitulated in organoids, the spatial relationships of the crypt-villus axis are not uniformly preserved. In addition, organisms are introduced in close proximity to crypt cells in the organoid, whereas in vivo they would need to traverse a greater distance from the bowel lumen to reach an anatomically equivalent location. The compact spatial organization also presents a technical challenge, in that there is a finite volume that can be injected into the organoid lumen, which could potentially be limiting for organisms that cannot be concentrated sufficiently. Second, we have shown that the organoid lumen is a discreet environment. We were unable to directly quantify the concentration of $\alpha$-defensins at basal levels or in response to stimuli in the organoid lumen. This issue is specific to defensins, as it is difficult to biochemically or histologically distinguish intracellular defensins stored in granules and those released into the lumen by degranulation, a mechanism well supported by the literature. ${ }^{11,31}$ A recent imaging study demonstrated secretion of lysozyme into the lumen of organoids over a time frame equivalent to that of our experiments. ${ }^{32}$ This supports our interpretation that $\alpha$-defensins are secreted even by unstimulated Paneth cells, as $\alpha$-defensins are stored in Paneth cell granules with lysozyme. ${ }^{4,11,33}$ Third, the degree to which the organoid lumen recapitulates the small intestinal lumen, particularly with regards to $\mathrm{pH}$ and osmolality, is unclear. The organoid lumen likely more accurately models the confines of intestinal crypts rather than the much larger bowel lumen. Fourth, although our study demonstrates that naturally secreted $\alpha$-defensins substantially contribute to inhibiting bacterial growth, it does not preclude a role for the microbiota and the immune system in impacting the phenotype of $M m p 7^{-1-}$ mice after enteric infection. ${ }^{7,8}$ Additionally, as $M m p 7^{-1-}$ mice lack both mature $\alpha$-defensins and cryptdin-related sequences, both of which are anti-bacterial, we cannot formally exclude a role for cryptdin-related sequences in the observed killing seen in WT organoids. ${ }^{34,35}$ Finally, we encountered a quantitative issue in some experiments owing to turbidity of the organoid medium $20 \mathrm{~h}$ postinfection in the $M m p 7^{-1-}$ cultures, most often with the 14028 s strain. We hypothesize that the absence of $\alpha$-defensins permitted bacterial overgrowth that compromised the integrity of the organoid lumen. This complication impacts the absolute quantification of experimental CFU but does not alter the qualitative interpretation of the experiments.

Overall, we believe that our organoid model has a significant potential for future applications. It is readily applicable to studying the direct impact of anti-microbial peptides and proteins on pathogen growth, which can likely be extended to viruses and parasites. Although we limited our assay to noninvasive strains of STM that are more sensitive to defensin killing, organoids could be used to interrogate invasive bacteria and their effects on host cells, and additional bacterial mutants could be studied alone or in combination. The veracity of the system can be further increased by inducing the differentiation of $\mathrm{M}$ cells and by co-culturing with immune cells. ${ }^{27,36}$ Finally, 
Table 1 Strains and plasmids used in the study

\begin{tabular}{|c|c|c|}
\hline $\begin{array}{l}\text { Strain or } \\
\text { plasmid } \\
\text { name }\end{array}$ & $\begin{array}{l}\text { Genotype or plasmid } \\
\text { backbone vector }\end{array}$ & Source \\
\hline BC155 & S. typhimurium LT2 & $\begin{array}{l}\text { Brad Cookson (University of } \\
\text { Washington, Seattle) }\end{array}$ \\
\hline $\mathrm{BC} 132$ & $\begin{array}{l}\text { S. typhimurium LT2 } \\
\text { phoP::Tn10Cam }\end{array}$ & $\begin{array}{l}\text { Brad Cookson (University of } \\
\text { Washington, Seattle) }\end{array}$ \\
\hline BC156 & S. typhimurium 14028s & $\begin{array}{l}\text { Brad Cookson (University of } \\
\text { Washington, Seattle) }\end{array}$ \\
\hline BC1563 & $\begin{array}{l}\text { S. typhimurium 14028s } \\
\text { fljBA::FRT sipB::FKF KanR }\end{array}$ & 41 \\
\hline SSW1 & BC156 sipB::FKF KanR & This study \\
\hline BC162 & BC156 phoP::Tn10Cam & $\begin{array}{l}\text { Brad Cookson (University of } \\
\text { Washington, Seattle) }\end{array}$ \\
\hline SSW3 & BC162 sipB::FKF KanR & This study \\
\hline pDW5 & pPtetA::GFP & 42 \\
\hline SSW4 & BC132 pDW5 & This study \\
\hline
\end{tabular}

human organoids can be cultured under similar conditions, permitting translational studies of acute infection and chronic diseases, such as inflammatory bowel disease. ${ }^{37}$ Thus the establishment of this model of enteric infection enables a broad range of studies of host-pathogen interactions.

\section{METHODS}

Ethics statement. All mouse experiments were performed in strict accordance with the Guide for the Care and Use of Laboratory Animals of the National Institutes of Health and following the International Guiding Principles for Biomedical Research Involving Animals. Protocols were approved by the Institutional Animal Care and Use Committee of the University of Washington.

Mice. WT and $M m p 7^{-1-}$ mice were on a C57BL/6NHsd background. ${ }^{38} \mathrm{Mmp}^{+/+} \mathrm{DEFA} 5^{+/-}$mice on a C57BL/6 background ${ }^{21}$ were obtained by cryorecovery from Jackson Laboratories and were interbred to yield $M m p 7^{+/+} D E F A 5^{+/+}$mice. $M m p 7^{+/+}$ $D E F A 5^{+/-}$mice were then backcrossed onto the $M m p 7^{-1-}$ background to yield $M m p 7^{-1-}$ DEFA5 $5^{+/-}$mice. NOD2 $2^{-1-}$ (stock number 5,763) and control WT C57BL/6J mice were obtained from Jackson Laboratories (Bar Harbor, ME). All mice were housed under specific pathogen-free conditions.

Bacterial strains and culture conditions. Mutants were generated in STM 14028s by P22 phage transduction. Strains were grown in LB-Miller at $37^{\circ} \mathrm{C}$ with aeration. Antibiotics were added at $50 \mu \mathrm{g} \mathrm{ml}^{-1}$. Strains and plasmids used are listed in Table $\mathbf{1 .}$

Small intestinal organoid production and maintenance. Organoids were cultured from crypt-enriched ileal fractions from 6-10-week-old WT and $M m p 7^{-1-}$ mice as previously described. ${ }^{1}$ Briefly, the distal $10 \mathrm{~cm}$ of the small intestine was removed and flushed with $0.04 \%$ sodium hypochlorite in PBS. After removal of mucus and villi, the epithelium was dissociated for $90 \mathrm{~min}$ at room temperature in a solution of $3 \mathrm{~mm}$ EDTA and $0.5 \mathrm{~mm}$ dithiothreitol in Hank's Buffered Salt solution (HBSS). Crypt-enriched fractions were identified following vigorous shaking into sequential changes of fresh, ice cold, sterile $\mathrm{Ca}^{2+} / \mathrm{Mg}^{2+}$-free HBSS. Cells were then concentrated by centrifugation at $300 \mathrm{~g}$ for $5 \mathrm{~min}$ at $4{ }^{\circ} \mathrm{C}$, and the pellet was resuspended in $300 \mu \mathrm{l}$ of HBSS containing $0.5 \mathrm{~mm}$ Rock inhibitor (Fisher, ThermoFisher Scientific, Waltham, MA) and $10 \mu \mathrm{M}$ Jagged-1
(AnaSpec, Fremont, CA). After a second round of centrifugation, the cell pellet was resuspended in growth factor reduced Matrigel (Corning, Tewksbury, MA). In all, $50 \mu \mathrm{l}$ aliquots were plated in the center of 24-well plates and overlayed with $500 \mu$ l of Complete Crypt Culture Medium (CCCM). ${ }^{10}$ Once established, culture media was supplemented with $200 \mu \mathrm{l}$ CCCM every 2-3 days. Organoids were subcultured every 6-7 days. ${ }^{10}$

Organoid microinjection and bactericidal assay. Organoids were subcultured as above 3-4 days prior to microinjection, deposited in $30 \mu$ l Matrigel on glass coverslips in 12-well tissue culture dishes, and overlaid with $1 \mathrm{ml}$ CCCM. Before injection, organoids on coverslips were washed twice with Advanced DMEM (Dulbecco's modified Eagle's medium)/F12 and overlaid with $1 \mathrm{ml}$ CCCM without antibiotics. A Nikon Ti microscope with Nomarski interference contrast (Nikon, Tokyo, Japan) fitted with a rotating glide stage and a FemtoJet Microinjector (Eppendorf, Hamburg, Germany) was used for microinjection. Injection needles were pulled from glass capillaries (1B100-4, World Precision Instruments, Sarasota, FL) on a horizontal bed puller (Sutter Instruments, Novato, CA). The tip ends were broken using tweezers.

For injection, overnight cultures of bacteria grown in LB-Miller broth were subcultured for an additional $2 \mathrm{~h}$ at $37^{\circ} \mathrm{C}$ with aeration under selection. One milliliter of subculture was washed twice and serially diluted in PBS to the desired concentration. The CFU of each inoculum was quantified from 20 injections into PBS prior to organoid injection.

To enumerate surviving CFU, media was removed from wells and centrifuged to recover bacteria, while organoids were removed from Matrigel using Cell Dissociation Solution (Corning) at $4{ }^{\circ} \mathrm{C}$ for $30 \mathrm{~min}$. Organoids in solution were added to the bacterial pellet, centrifuged at $300 \mathrm{~g}$ for $5 \mathrm{~min}$ and resuspended in $100 \mu \mathrm{l}$ sterile water for $5 \mathrm{~min}$ to lyse eukaryotic cells. The sample was then vortexed for $15 \mathrm{~s}$ before serial dilution in PBS and plating on LB agar plates. Plates were incubated at $37^{\circ} \mathrm{C}$, and colonies were counted after $18-20 \mathrm{~h}$ to determine CFU.

Twenty organoids per sample were injected one time each with STM. Each injection contained $5 \times 10^{4} \mathrm{CFU}$ for Figure $1 \mathrm{~b} ; 5 \times 10^{3}$ CFU for Figures $\mathbf{2 a}-\mathbf{e}, \mathbf{5 b}, \mathbf{c}, \mathbf{6 b}, \mathbf{c}$, and Supplementary Figure S1; and $50 \mathrm{CFU}$ for Figure 4a,b. Note that we have graphed the total inoculum delivered to the entire well in all the figures except in Figure 2d and Supplementary Figure S1D in which the inoculum delivered to a single organoid is graphed. Samples were then incubated in CCCM without antibiotics ( $1 \mathrm{~h}$ for Figures $\mathbf{5 b}, \mathbf{c}, \mathbf{6 b}, \mathbf{c}$, and Supplementary Figure $\mathrm{S} 1$ or $2 \mathrm{~h}$ for Figures $\mathbf{1 b}, \mathbf{2 a}-\mathbf{e}$, and $\mathbf{4 a}, \mathbf{b}$ ) and then for an equal amount of time with $100 \mu \mathrm{g} \mathrm{ml}^{-1}$ gentamicin. Where indicated, $10 \mu \mathrm{M}$ carbamylcholine chloride (Sigma-Aldrich, St. Louis, MO) was added to the culture well $30 \mathrm{~min}$ before injection. For Figure 1b, CFU were enumerated at this point. For subsequent experiments, injected organoids were washed twice with Advanced DMEM/F12 and grown in CCCM without antibiotics. Surviving CFU was enumerated at 0, 5, $7,9,16$, or $20 \mathrm{~h}$ postinjection, as indicated in each figure legend.

Image analysis. Epifluorescence images were acquired using a Nikon Ti-E inverted microscope fitted with a $\times 40$ objective, a CCD camera, and image acquisition software (NIS Elements, Nikon). In all, $4-\mu \mathrm{m}$ $z$-stacks were obtained in both bright field and fluorescent channels for each organoid. Image analysis was performed using Image (NIH, Bethesda, MD) v.1.45s. ${ }^{39}$ To account for autofluorescence, a threshold was determined using an average of 10 uninjected organoids, and the integrated density of signal above threshold for the most focused plane of each organoid was then calculated.

Immunohistochemistry. Small intestinal organoids were removed from Matrigel using Cell Dissociation Solution as above, concentrated by centrifugation, and fixed in $10 \%$ neutral buffered formalin. Fixed organoids were then resuspended in Histogel (ThermoFisher Scientific), stored overnight in $10 \%$ neutral buffered formalin, and embedded in paraffin. Deparaffinized samples were incubated with 
goat anti-Crp5 (1:8,000, a kind gift from Dr Andre Ouellette $\left.{ }^{35}\right)$ or goat immunoglobulin $\mathrm{G}\left(0.6 \mu \mathrm{g} \mathrm{ml}{ }^{-1}\right.$, Life Technologies, Grand Island, NY) followed by diaminobenzidine precipitation and hematoxylin counterstaining. Non-sequential sections from the same blocks were stained with hematoxylin and eosin.

Western blotting. Small intestinal organoids were cultured for 6 days and removed from Matrigel using Cell Dissociation Solution as above. Cell lysates were prepared in radio-immunoprecipitation assay buffer (50 mM Tris- $\mathrm{HCl}, 150 \mathrm{~mm} \mathrm{NaCl}, 1 \mathrm{~mm}$ EDTA, 1\% NP-40, $0.5 \%$ deoxycholate, $0.1 \%$ sodium dodecyl sulfate) with protease inhibitors. After separation by sodium dodecyl sulfate PAGE, immunoblots were probed for MMP7 (ab5706, Abcam, Cambridge, MA) or GAPDH (sc-32233, Santa Cruz, CA) and visualized with chemiluminescence. To generate rMMP7, the Mmp7 gene was cloned into a pET27 vector with a C-terminal Strep tag. Upon expression in E. coli strain BL21, rMMP7 was purified from solubilized inclusion bodies by affinity chromatography using StrepTactin Sepharose (IBA GmbH, Goettingen, Germany).

AU-PAGE and AU-PAGE western blotting. Organoids or crypt-enriched fractions from mouse ileum were concentrated by centrifugation, resuspended in $30 \%$ acetic acid, and sonicated. After incubation overnight at $4{ }^{\circ} \mathrm{C}$ with agitation, samples were diluted threefold with water. Insoluble material was removed by centrifugation at $100,000 \mathrm{~g}$ for $2 \mathrm{~h}$ at $4{ }^{\circ} \mathrm{C}$, protein concentrations of the supernatants were determined by Bio-Rad Protein Assay (Bio-Rad, Hercules, CA), and equivalent amounts of each sample were lyophilized. Lyophilized samples were dissolved in 5\% acetic acid and separated by $17 \%$ AU-PAGE. ${ }^{14}$ Folded Crp23 was created from a synthesized $80 \%$ pure linear peptide (CPC Scientific, Sunnyvale, CA) by the same procedure as previously reported for the $\alpha$-defensin HD5. ${ }^{40}$ Proteins were visualized with SYPRO Ruby (Life Technologies). Gels were imaged using a Typhoon 9,400 variable mode imager (GE Healthcare, Piscataway, NJ). For western blotting, samples were separated by 12.5\% AU-PAGE and semi-dry transferred to nitrocellulose membranes. Membranes were immediately fixed in glutaraldahyde and blocked in 5\% milk, before overnight incubation at room temperature in rabbit anti-HD5 antibody (kind gift from Edith Porter ${ }^{12}$ ) at a 1:1,000 dilution. Membranes were incubated in goat-anti-rabbit Alexa Fluor 488 (Life Technologies) and imaged using a Typhoon 9,400 variable mode imager (GE Healthcare).

Statistics. Experiments were analyzed using Prism (v. 5.0d, GraphPad, La Jolla, CA). For Figure 1b, data were log transformed and analyzed by one-way analysis of variance with Tukey's post-tests. For Figure 2c, one-way analysis of variance with Bonferroni's Multiple Comparison Test was used. For Figures 2d,e, 5b,c, and $\mathbf{6 b}, \mathbf{c}$, and Supplementary Figures S1C,D and S2B,C, data were log transformed and analyzed by repeated-measures one-way analysis of variance with Tukey's post-tests. For Figure 4a,b, data were log transformed and analyzed by paired $t$-test. In all the analyses, $P<0.05$ was considered significant. In the figures, single asterisk $\left(^{*}\right)$ indicates $P<0.05$, double asterisks ${ }^{* *}$ ) indicates $P<0.01$, and triple asterisks ${ }^{* * *}$ ) indicates $P<0.005$.

SUPPLEMENTARY MATERIAL is linked to the online version of the paper at http://www.nature.com/mi

\section{ACKNOWLEDGMENTS}

We thank B. Thomasson and J. Maxwell for NOD2 organoid isolation; M. Stewart, L. Cummings, and B. Cookson for STM strains and plasmids; and M. Stewart for help in STM strain construction. J. Mougous and M. LeRoux provided access and technical expertise for microcopy, and D. Miller and J. Horsman helped establish a microinjection system. R. Edwards and K. Wang provided guidance on establishing organoid cultures. This work was supported in part by the National Institutes of Health grants R56 Al097311 (to J.G.S.), R01 Al104920 (to J.G.S.), HL098067 (to W.C.P.), T32 GM07270 (to S.S.W.), and T32 Al083203 (to S.S.W.).

\section{AUTHOR CONTRIBUTIONS}

S.S.W. and J.G.S. conceived and designed the experiments. S.S.W., A.T., and M.K.H. performed the experiments. S.S.W., M.K.H., and J.G.S. analyzed the data. S.S.W., J.G.S., A.T., and W.C.P. wrote the paper.

\section{DISCLOSURE}

The authors declared no conflict of interest.

(c) 2015 Society for Mucosal Immunology

\section{REFERENCES}

1. Sato, T. et al. Single Lgr5 stem cells build crypt-villus structures in vitro without a mesenchymal niche. Nature 459, 262-265 (2009).

2. Sato, T. \& Clevers, H. Growing self-organizing mini-guts from a single intestinal stem cell: mechanism and applications. Science 340, 1190-1194 (2013).

3. Miyoshi, H., Ajima, R., Luo, C.T., Yamaguchi, T.P. \& Stappenbeck, T.S. Wnt5a potentiates TGF-beta signaling to promote colonic crypt regeneration after tissue injury. Science 338, 108-113 (2012).

4. Ayabe, T. et al. Secretion of microbicidal alpha-defensins by intestinal Paneth cells in response to bacteria. Nat. Immunol. 1, 113-118 (2000).

5. Bevins, C.L. \& Salzman, N.H. Paneth cells, antimicrobial peptides and maintenance of intestinal homeostasis. Nat. Rev. Microbiol. 9, 356-368 (2011).

6. Bowdish, D.M., Davidson, D.J. \& Hancock, R.E. Immunomodulatory properties of defensins and cathelicidins. Curr. Top. Microbiol. Immunol. 306, 27-66 (2006).

7. Salzman, N.H. et al. Enteric defensins are essential regulators of intestinal microbial ecology. Nat. Immunol. 11, 76-83 (2010).

8. Wilson, C.L. et al. Regulation of intestinal alpha-defensin activation by the metalloproteinase matrilysin in innate host defense. Science 286, 113-117 (1999).

9. Wehkamp, J. et al. Reduced Paneth cell alpha-defensins in ileal Crohn's disease. Proc. Natl. Acad. Sci. USA 102, 18129-18134 (2005).

10. Sato, T. \& Clevers, H. Primary mouse small intestinal epithelial cell cultures. Methods Mol. Biol. 945, 319-328 (2013).

11. Clevers, H.C. \& Bevins, C.L. Paneth cells: maestros of the small intestinal crypts. Annu. Rev. Physiol. 75, 289-311 (2013).

12. Porter, E.M., Liu, L., Oren, A., Anton, P.A. \& Ganz, T. Localization of human intestinal defensin 5 in Paneth cell granules. Infect. Immun. 65, 2389-2395 (1997).

13. Shanahan, M.T., Tanabe, H. \& Ouellette, A.J. Strain-specific polymorphisms in Paneth cell alpha-defensins of C57BL/6 mice and evidence of vestigial myeloid alpha-defensin pseudogenes. Infect. Immun. 79, 459-473 (2011).

14. Mastroianni, J.R. et al. Alternative luminal activation mechanisms for paneth cell alpha-defensins. J. Biol. Chem. 287, 11205-11212 (2012).

15. Miller, S.I., Pulkkinen, W.S., Selsted, M.E. \& Mekalanos, J.J. Characterization of defensin resistance phenotypes associated with mutations in the phoP virulence regulon of Salmonella typhimurium. Infect. Immun. 58, 3706-3710 (1990).

16. Lara-Tejero, M. \& Galan, J.E. Salmonella enterica serovar typhimurium pathogenicity island 1-encoded type III secretion system translocases mediate intimate attachment to nonphagocytic cells. Infect. Immun. 77, 2635-2642 (2009).

17. Salzman, N.H., Ghosh, D., Huttner, K.M., Paterson, Y. \& Bevins, C.L. Protection against enteric salmonellosis in transgenic mice expressing a human intestinal defensin. Nature 422, 522-526 (2003).

18. Ghosh, D. et al. Paneth cell trypsin is the processing enzyme for human defensin-5. Nat. Immunol. 3, 583-590 (2002).

19. Philpott, D.J., Sorbara, M.T., Robertson, S.J., Croitoru, K. \& Girardin, S.E. NOD proteins: regulators of inflammation in health and disease. Nat. Rev. Immunol. 14, 9-23 (2014).

20. Petnicki-Ocwieja, T. et al. Nod2 is required for the regulation of commensal microbiota in the intestine. Proc. Natl. Acad. Sci. USA 106, 15813-15818 (2009).

21. Biswas, A. et al. Induction and rescue of Nod2-dependent Th1-driven granulomatous inflammation of the ileum. Proc. Natl. Acad. Sci. USA 107, 14739-14744 (2010). 
22. Kobayashi, K.S. et al. Nod2-dependent regulation of innate and adaptive immunity in the intestinal tract. Science 307, 731-734 (2005).

23. Shanahan, M.T. et al. Mouse Paneth cell antimicrobial function is independent of Nod2. Gut 63, 903-910 (2013).

24. Selsted, M.E. \& Ouellette, A.J. Mammalian defensins in the antimicrobial immune response. Nat. Immunol. 6, 551-557 (2005).

25. Lehrer, R.I. \& Lu, W. alpha-Defensins in human innate immunity. Immunol. Rev. 245, 84-112 (2012).

26. Tsilingiri, K. et al. Probiotic and postbiotic activity in health and disease: comparison on a novel polarised ex-vivo organ culture model. Gut 61, 1007-1015 (2012).

27. Koo, B.K. et al. Controlled gene expression in primary Lgr5 organoid cultures. Nat. Methods 9, 81-83 (2012).

28. Sun, L., Nava, G.M. \& Stappenbeck, T.S. Host genetic susceptibility, dysbiosis, and viral triggers in inflammatory bowel disease. Curr. Opin. Gastroenterol. 27, 321-327 (2011).

29. Simms, L.A. et al. Reduced alpha-defensin expression is associated with inflammation and not NOD2 mutation status in ileal Crohn's disease. Gut 57, 903-910 (2008).

30. Nigro, G., Rossi, R., Commere, P.H., Jay, P. \& Sansonetti, P.J. The cytosolic bacterial peptidoglycan sensor nod2 affords stem cell protection and links microbes to gut epithelial regeneration. Cell Host Microbe 15, 792-798 (2014).

31. Porter, E.M., Bevins, C.L., Ghosh, D. \& Ganz, T. The multifaceted Paneth cell. Cell. Mol. Life Sci. 59, 156-170 (2002).

32. Schwank, G., Andersson-Rolf, A., Koo, B.K., Sasaki, N. \& Clevers, H. Generation of BAC transgenic epithelial organoids. PLoS One 8, e76871 (2013).

33. Putsep, K. et al. Germ-free and colonized mice generate the same products from enteric prodefensins. J. Biol. Chem. 275, 40478-40482 (2000).
34. Hornef, M.W., Putsep, K., Karlsson, J., Refai, E. \& Andersson, M. Increased diversity of intestinal antimicrobial peptides by covalent dimer formation. Nat. Immunol. 5, 836-843 (2004).

35. Shanahan, M.T. et al. Elevated expression of paneth cell CRS4C in ileitisprone samp1/YitFc mice: regional distribution, subcellular localization, and mechanism of action. J. Biol. Chem. 285, 7493-7504 (2010).

36. de Lau, W. et al. Peyer's patch M cells derived from Lgr5(+) stem cells require SpiB and are induced by RankL in cultured "miniguts". Mol. Cell Biol. 32, 3639-3647 (2012).

37. Sato, T. et al. Long-term expansion of epithelial organoids from human colon, adenoma, adenocarcinoma, and Barrett's epithelium. Gastroenterology 141, 1762-1772 (2011).

38. Kassim, S.Y. et al. Individual matrix metalloproteinases control distinct transcriptional responses in airway epithelial cells infected with Pseudomonas aeruginosa. Infect. Immun. 75, 5640-5650 (2007).

39. Rasband, W.S. ImageJ. http://rsb.info.nih.gov/ij/ (U.S. National Institutes of Health, Bethesda, MD, USA, 1997-2014).

40. Gounder, A.P., Wiens, M.E., Wilson, S.S., Lu, W. \& Smith, J.G. Critical determinants of human alpha-defensin 5 activity against non-enveloped viruses. J. Biol. Chem. 287, 24554-24562 (2012).

41. Stewart, M.K., Cummings, L.A., Johnson, M.L., Berezow, A.B. \& Cookson, B.T. Regulation of phenotypic heterogeneity permits Salmonella evasion of the host caspase-1 inflammatory response. Proc. Natl. Acad. Sci. USA 108, 20742-20747 (2011).

42. Cummings, L.A., Wilkerson, W.D., Bergsbaken, T. \& Cookson, B.T. In vivo, flic expression by Salmonella enterica serovar Typhimurium is heterogeneous, regulated by ClpX, and anatomically restricted. Mol. Microbiol. 61, 795-809 (2006). 